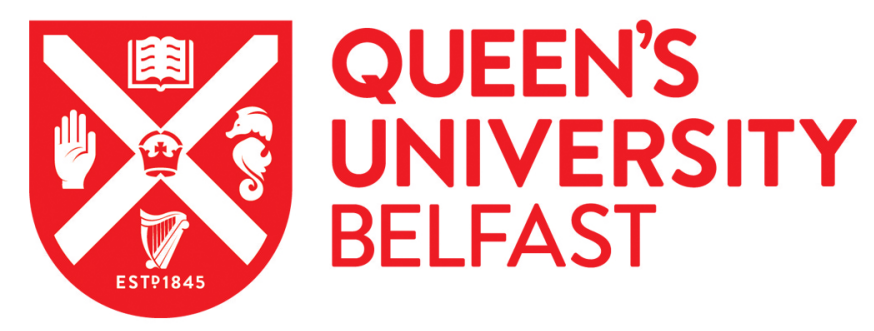

\title{
Coexistence of negative and positive polarity electrostatic solitary waves in ultradense relativistic negative-ion-beam permeated plasmas
}

Elkamash, I. S., \& Kourakis, I. (2018). Coexistence of negative and positive polarity electrostatic solitary waves in ultradense relativistic negative-ion-beam permeated plasmas. Physics of Plasmas, 25(5), 1-17. [052124]. https://doi.org/10.1063/1.5024519

Published in:

Physics of Plasmas

Document Version:

Peer reviewed version

Queen's University Belfast - Research Portal:

Link to publication record in Queen's University Belfast Research Portal

Publisher rights

Copyright 2018 AIP Publishing. This work is made available online in accordance with the publisher's policies. Please refer to any applicable terms of use of the publisher.

\section{General rights}

Copyright for the publications made accessible via the Queen's University Belfast Research Portal is retained by the author(s) and / or other copyright owners and it is a condition of accessing these publications that users recognise and abide by the legal requirements associated with these rights.

Take down policy

The Research Portal is Queen's institutional repository that provides access to Queen's research output. Every effort has been made to ensure that content in the Research Portal does not infringe any person's rights, or applicable UK laws. If you discover content in the Research Portal that you believe breaches copyright or violates any law, please contact openaccess@qub.ac.uk. 


\title{
Coexistence of negative and positive polarity electrostatic solitary waves in ultradense relativistic negative-ion-beam permeated plasmas
}

\author{
I. S. Elkamash ${ }^{1,2, *}$ and I. Kourakis ${ }^{1, \dagger}$ \\ 1 Centre for Plasma Physics, Queen's University Belfast, \\ Belfast BT7 1NN, Northern Ireland, UK \\ 2 Physics Department, Faculty of Science, \\ Mansoura University, 35516 Mansoura, Egypt
}

\begin{abstract}
The criteria for occurrence and the dynamical features of electrostatic solitary waves (ESWs) in a homogeneous, unmagnetized ultradense plasma penetrated by a negative ion beam are investigated, relying on a quantum hydrodynamic model. The ionic components are modeled as inertial fluids, while the relativistic electrons obey Fermi-Dirac statistics. A new set of exact analytical conditions for localized solitary pulses to exist are obtained, in terms of the plasma density. The algebraic analysis reveals that these depend sensitively on the negative ion beam characteristics, that is, the beam velocity and density. Particular attention is paid to the simultaneous occurrence of positive and negative potential pulses, identified by their respective distinct ambipolar electric field structure forms. It is shown that coexistence of positive and negative potential pulses occurs in a certain interval of parameter values, where the ion beam inertia becomes significant.
\end{abstract}

\section{INTRODUCTION}

Electrostatic solitary waves (ESWs), i.e. localized excitations manifested as localized pulse-shaped potential structures associated with characteristically shaped bipolar electric-field forms and copropagating plasma density disturbances, are not only tacitly observed in laboratory experiments $[1,2]$, but are also ubiquitous in Space plasmas [3]. Following the seminal work of R.Z. Sagdeev in the 1960s [4], these have been effective modelled by an integrable (pseudo-Hamiltonian) model [5], which was later generalized to take into account different plasma configurations or/and other generic types of electrostatic structures [6]. ESWs in ultrahigh density plasmas permeated by a negative-ion beam will be the focus of this study. Plasmas containing a fraction of negative ions possess characteristic dynamical properties that may differ substantially from those of "traditional" (textbook) electronion plasmas. Such plasmas have been generated in the laboratory by using a Q-machine [7, 8], a double plasma device [9-11], electron cyclotron resonance (ECR) based plasma devices $[12,13]$ and specially designed discharge chambers [14]. Negative ion plasmas (NIP) have also been observed in Space environments, e.g. in the D and F regions of the Earths ionosphere $[15,16]$ and in the inner coma of comet Halley [17]. Negative ion plasmas are used in industrial applications, such as neutral beam injection relying on a negative ion source to achieve plasma heating in plasma etching [18], in material processing [19] and in fusion reactors [20].

Wong et al [21] produced a collisionless plasma with negative ions $\left(S F_{6}^{-}\right)$in the laboratory and they observed a

\footnotetext{
*Email address: elkamashi@gmail.com

${ }^{\dagger}$ www.kourakis.eu; email address: IoannisKourakisSci@gmail.com
}

supersonic fast ion mode due to out phase oscillations between positive and negative ions. By using the reductive perturbation technique, Das [22] studied the effect of critical plasma concentration of the negative ions on wavebreaking of ion acoustic waves in collisionless plasma. Ludwig et al [23] experimentally showed that applying a neative voltage pulse on multi-component plasma with sufficient large negative ion concentration may lead to compression of the negative ion density and formation of a rarefactive solitary wave (a pulse). On the other hand, Nakamura et al [24] demonstrated that, at critical concentration of the negative ions in the plasma, compressive and rarefactive solitons may (co-)exist in the laboratory and they compared the observed soliton with the soliton solution of the modified Korteweg-de Vries (mKdV) equation. Independently, based on a stability analysis of the nonlinear Schrodinger equation (NLSE), the modulation stability profile of ion acoustic waves in collisionless plasmas with negative ions was investigated theoretically, and either bright or dark soliton have been shown that to exist, depending on the concentration of the negative ions[25]. The existence of ion acoustic solitons in low $\beta$ magnetized plasma with negative ions has been established theoretically by means of a Zakharov Kuznetsov (ZK) equation [26]. By using a triple plasma device, the formation of strong double layers in multi-component plasmas with a significant concentration of negative ions has been examined in the laboratory [27]. Depending on the ratio of negative to positive ions, either compressive or rarefactive collisionless ion acoustic shock waves were experimentally observed in a plasma formed in a single ended Q - machine [28]. Sharma et al [29] showed that the propagation of an ion beam through a magnetized plasma with negative ions can drive an ion acoustic wave instability via Cerenkov type beam-plasma interaction. Solitary wave polarity reversal (i.e., generation of rarefactive, rather than compressive excitations) in a negative 
ion-beam permeated plasma was observed in the laboratory, by means of a double plasma device [30]. In an similar study, the impact of negative ions on drift wave propagation was investigated by means of the DUSTWHEEL experiment in Kiel [31], where the negative ion density was shown to affect the dispersion characteristics and the stability profile of drift waves. The influence of negative ions on drift ion wave instability in a weakly collisional magnetized plasma was investigated by using linear kinetic theory in Ref. 32. A year ago, the so called critical plasma concentration was invoked for negative ions, in order to study the nonlinear evolution of ionacoustic envelope wavepackets and to establish the existence of second-order ion-acoustic "Peregrine solitons" (breathers) experimentally [33]. More recently, the different vibration modes occurring in negative-ion plasma in the presence of a streaming ion beam were investigated by means of one-dimensional electrostatic particle in cell (PIC) computer simulations [34].

Ultrahigh density plasma configurations are encountered in various physical contexts, including compact astrophysical objects, nanometer size solid metals, and lasercompressed plasma [35-37]. Quantum effects are relevant in ultrahigh density astrophysical objects, e.g. in pulsars, in blazars and in supernova-driven plasma flows. For instance, white dwarfs, whose centers are characterized of a density and temperature of the order of $n_{0}=1.5 \times 10^{32} \mathrm{~m}^{-3}$ and $T=10^{7} \mathrm{~K}$, respectively [38]. Electrons in solids are always degenerate and behave quantum mechanically $[39,40]$. For example, the density of metallic gold at room temperature $T=300 \mathrm{~K}$ is $n_{0}=5.9 \times 10^{28} \mathrm{~m}^{-3}[40]$, while in GaAs semiconductor it is $n_{0}=4.7 \times 10^{22} \mathrm{~m}^{-3}$ [41]. Ultrahigh density plasma also occurs in the context of inertial confinement fusion, where an ultraintense laser beam irradiates a solid target, whose density is compressed up to 1000 times its solid density, corresponding to a number density of roughly $n_{0}=10^{32} \mathrm{~m}^{-3}$, a fact imposing a quantum treatment in modeling [42].

It is by now established that relativistic ion beams may occur in extreme plasma environments, such as the ones mentioned above [43-45]. In Ref. 46, Sauer et al argued, on the basis of a Hall-MHD description, that ion beams may play a significant role in the existence of quasi-steady nonlinear soliton-like structures in cometary tails and in the magnetospheres of unmagnetized planets, such as Venus and Mars. Using of a fully relativistic electromagnetic particle-in-cell (PIC) code, Dieckmann et al $[47,48]$ have shown that the interaction of a relativistic ion beam with plasma can generate intense electrostatic solitary waves which can be responsible for the acceleration of electrons to ultrahigh energies in supernova remnant blast shells. Recently, Deka et al [49] investigated the effect of an relativistic ion beam on the existence and propagation of ion acoustic solitary waves in a relativistic degenerate magnetized plasma.

For the sake of rigor, let us point out that we have adopted a collisionless plasma model, adopted to describe a high particle-density configuration, also taking into account relativistic electrons effects. Inter-particle interactions (coupling) are therefore assumed to be weak. According to the discussion in Ref. 50, one may consider the quantum coupling parameter $\Gamma_{Q}^{3 / 2}=\frac{1}{n_{0} \lambda_{F}^{3}}$, where $n_{0}$ is the electron number density, $\lambda_{F}=\frac{V_{F}}{\omega_{p}}$ is the Fermi screening length and $V_{F}=\sqrt{T_{F} / m_{e}}$ is the Fermi velocity, while $T_{F}$ denotes the Fermi temperature. Accordingly, for weakly-coupled plasmas, one considers $\Gamma_{Q} \ll 1$, where inter-particle coupling is weak, within a collisionless plasma model. For instance, at room temperature $T=300 K$, the typical values of electron parameters in metals are $n_{0} \approx 10^{29} \mathrm{~m}^{-3}, V_{F} \approx 10^{6} \mathrm{~ms}^{-1}, \omega_{p} \approx 10^{16} \mathrm{~s}^{-1}$ and $\lambda_{F} \approx 10^{-10} \mathrm{~m}$, therefore $\Gamma_{Q} \approx 1$ (order of magnitude). This means one should take into account the effect of collisions. In our case, however, the Pauli exclusion principle decreases the collision rate dramatically in our time scale of interest. The average collision frequency is given by $\nu_{e e}=\frac{K_{B} T^{2}}{\hbar T_{F}}$, so the $\frac{\nu_{e e}}{\omega_{p}}=\frac{1}{\Gamma_{Q}^{1 / 2}}\left(\frac{T}{T_{F}}\right)^{2} \ll 1$, which is relevant in degenerate electrons plasma. Consequently, the time scale for electron ion collision $\tau_{e i}=10^{-10} \mathrm{~s}$ is much greater than the plasma period $\tau_{p} \approx \omega_{p}^{-1}=10^{-16} s$, i.e. $\tau_{e i} \gg \tau_{p}$. Furthermore, the electron ion collisions rate is $\tau_{e i} \approx 10^{-14} s$ which is much larger than $\tau_{p}$ too. So, our model suggests that electrostatic solitary waves will survive over long times, i.e. a number of plasma periods, but nonetheless on a finite timescale, in respect of the ordering $\tau_{p}<\tau_{e i} \ll \tau_{e e}$. Remarkably, the effect of injection of a positive ion beam on the existence of large amplitude ion acoustic solitary waves of both positive and negative polarity (i.e., both compressive and rarefactive pulses) in low density plasma was investigated experimentally, and these structures were observed in the laboratory [51]. Our aim in this article is to investigate the conditions for the coexistence of positive and negative potential pulses and the characteristics of electrostatic (ES) localized modes (solitary waves) propagating in an ultradense electron-ion plasma permeated by a tenuous negative-ion beam.

Based on the analytical model for relativistic electrostatic excitations introduced in Refs. 52-54, here generalized to incorporate a negative-ion drifting fluid (the beam), we propose a multifluid relativistic model for electrostatic plasma excitations. The model is used as basis for a nonlinear analysis, in search of stationary profile localized solutions (solitary waves).

The layout of this article goes as follows. The analytical setting is presented in Section II and the inherent limitations of the model are discussed. Nonlinear dynamical system analysis based on a pseudopotential methodology is carried out in Section III, which provides analytical expressions for the state variables, expressed in terms of a propagating potential excitation. We show that pulse occurrence is only possible for certain parameter regions, which are investigated in Section IV. It is shown that either positive or negative potential excitations may exist, and these may even occur simultaneously, in a given re- 
gion of parameter values. A parametric analysis follows, in Section V, elucidating the dependence of electrostatic pulse characteristics on the beam properties and other intrinsic plasma parameters. Our findings are finally summarized in the concluding Section VI.

\section{THE MODEL}

A three-component plasma is considered, consisting of a dominant ion population (mass $m_{i}$, positive charge $\left.q_{i}=+Z_{i} e\right)$, a secondary - negatively charged - ion species, representing a tenuous beam (mass $m_{b}$, charge $q_{b}=-Z_{b} e$ ) and electrons (mass $m_{e}$, charge $-e$ ); $e$ denotes the elementary (absolute) charge, as usual. Spatial variation of the plasma plasma state variables is assumed to occur only in the longitudinal direction, hence the plasma dynamics can be described by a one-dimensional (1D) geometry for simplicity. Our study relies on a multifluid approach, to be introduced in the following paragraph. The total current is thought to be negligible, so that magnetization can be ignored (electrostatic approximation, implying a tenuous beam). This description builds upon a relativistic model for electrostatic introduced earlier [52]-[53], in fact generalizing the framework proposed therein to accommodate a drifting negative ion component (the beam) [56].

The main ion population is modeled classically as a "cold ion" fluid, for simplicity, given the large ion mass, compared to the electrons. The continuity and momentum equations of motion for the ion fluid respectively read:

$$
\begin{aligned}
& \frac{\partial\left(\gamma_{i} n_{i}\right)}{\partial t}+\frac{\partial}{\partial x}\left(\gamma_{i} n_{i} u_{i}\right)=0, \\
& \frac{\partial\left(\gamma_{i} u_{i}\right)}{\partial t}+u_{i} \frac{\partial\left(\gamma_{i} u_{i}\right)}{\partial x}=-\frac{e Z_{i}}{m_{i}} \frac{\partial \phi}{\partial x},
\end{aligned}
$$

where we have defined the electron charge $e$, the ion charge (state) $Z_{i}$, and the ion mass $m_{i}$. The dynamical variables $n_{i}$ and $u_{i}$ denote the ion fluid density and speed, respectively. One recognizes the electrostatic force $e Z_{i} E$ in the right-hand side (RHS) of the momentum equation, where $E=-\partial \phi / \partial x$ is the electric field deriving from an electrostatic potential function $\phi$.

The electron fluid obeys the equations [52]:

$$
\begin{gathered}
\frac{\partial\left(\gamma_{e} n_{e}\right)}{\partial t}+\frac{\partial}{\partial x}\left(\gamma_{e} n_{e} u_{e}\right)=0 \\
\sqrt{1+\xi^{2}}\left[\frac{\partial\left(\gamma_{e} u_{e}\right)}{\partial t}+u_{e} \frac{\partial\left(\gamma_{e} n_{e}\right)}{\partial x}\right]= \\
\frac{e}{m_{e}} \frac{\partial \phi}{\partial x}-\frac{\gamma_{e}}{n_{e} m_{e}}\left(\frac{\partial P_{e}}{\partial x}+\frac{u_{e}}{c^{2}} \frac{\partial P_{e}}{\partial t}\right),
\end{gathered}
$$

where the electron fluid density and speed variables are denoted by $n_{e}$ and $u_{e}$. In the above relations, $m_{e}$ is the electron mass at rest, $c$ is the speed of light in vacuum and $h$ is Planck's constant,

In the case of very high density plasmas, quantum effects may become important. Degeneracy effects in particular exceed the (classical) thernal pressure and even the quantum (Bohm type) pressure, for higher density [57]. A Fermi-Dirac distribution describes the electron statistics in these conditions. A new equation of state then needs to be taken into account; cf. the last term in the RHS of Eq. (4). Here, we have adopted the quantum relativistic equation of state adopted in Ref. 52:

$$
P_{e}=\frac{2 m_{e}^{2} c^{3}}{h}\left[\xi\left(1+\xi^{2}\right)^{1 / 2}-\sinh ^{-1} \xi\right],
$$

where the parameter $\xi=p_{F e} / m_{e} c=h n_{e} /\left(4 m_{e} c\right)$ is related to the electronic density (the classical picture is recovered, as expected, in the limit $h \rightarrow 0$ ). We also note in (4) the appearance of the electrostatic force term, associated with the gradient of a potential function $\phi$.

The negative ion beam (fluid) is described by:

$$
\begin{aligned}
& \frac{\partial\left(\gamma_{b} n_{b}\right)}{\partial t}+\frac{\partial}{\partial x}\left(\gamma_{b} n_{b} u_{b}\right)=0 \\
& \frac{\partial\left(\gamma_{b} u_{b}\right)}{\partial t}+u_{b} \frac{\partial\left(\gamma_{b} u_{b}\right)}{\partial x}=\frac{e Z_{b}}{m_{b}} \frac{\partial \phi}{\partial x},
\end{aligned}
$$

where $m_{b}$ is the beam ion mass, $n_{b}$ is the beam ion fluid density and $u_{b}$ is the beam ion fluid speed. The relativistic factor $\gamma_{j}=1 / \sqrt{1-u_{j}^{2} / c^{2}}$ (for $\left.j=i, e, b\right)$ appearing in the model equations stems from Lorentz transformations and from the resulting relations among different state variables between inertial frames, as discussed in Ref. 52; also cf. [55]. The equilibrium state (denoted by subscript ' 0 ') is defined by the state "vector" $\left.\left(n_{i}, u_{i} ; n_{b}, u_{b} ; n_{e}, u_{e}\right)\right|_{0}=\left(n_{i 0}, 0 ; n_{b, 0}, u_{b 0} ; n_{e 0}, 0\right)$, where $u_{b 0}$ is the unperturbed beam fluid speed.

Poisson's equation reads:

$$
\frac{\partial^{2} \phi}{\partial x^{2}}=\frac{e}{\epsilon_{0}}\left(\gamma_{e} n_{e}-\gamma_{i} Z_{i} n_{i}+\gamma_{b} Z_{b} n_{b}\right),
$$

where $\epsilon_{0}$ is the permittivity of vacuum. Charge neutrality at equilibrium (only) implies: $n_{e 0}-Z_{i} n_{i 0}+\gamma_{b 0} Z_{b} n_{b 0}=0$, where the index ' 0 ' (in $\gamma_{b 0}=1 / \sqrt{1-u_{b 0}^{2} / c^{2}}, n_{e 0}, n_{i 0}$ and $n_{b 0}$ ) denotes the equilibrium values of the respective variables.

The system of Eqs. (1)-(8) can be cast in the (dimensionless) form:

$$
\begin{aligned}
& \frac{\partial\left(\gamma_{i} n_{i}\right)}{\partial t}+\frac{\partial}{\partial x}\left(\gamma_{i} n_{i} u_{i}\right)=0, \\
& \frac{\partial\left(\gamma_{i} u_{i}\right)}{\partial t}+u_{i} \frac{\partial\left(\gamma_{i} u_{i}\right)}{\partial x}=-\frac{\partial \phi}{\partial x}, \\
& \frac{\partial\left(\gamma_{e} n_{e}\right)}{\partial t}+\frac{\partial}{\partial x}\left(\gamma_{e} n_{e} u_{e}\right)=0, \\
& \frac{\partial\left(\gamma_{e} u_{e}\right)}{\partial t}+u_{e} \frac{\partial\left(\gamma_{e} n_{e}\right)}{\partial x}= \\
& \frac{1}{H \mu_{e}} \frac{\partial \phi}{\partial x}-\frac{n_{e} \gamma_{e}}{H^{2} \mu_{e}}\left(\frac{\partial n_{e}}{\partial x}+\alpha u_{e} \frac{\partial n_{e}}{\partial t}\right), \\
& \frac{\partial\left(\gamma_{b} n_{b}\right)}{\partial t}+\frac{\partial}{\partial x}\left(\gamma_{b} n_{b} u_{b}\right)=0, \\
& \frac{\partial\left(\gamma_{b} u_{b}\right)}{\partial t}+u_{b} \frac{\partial\left(\gamma_{b} u_{b}\right)}{\partial x}=\frac{1}{\mu_{b}} \frac{\partial \phi}{\partial x}, \\
& \frac{\partial^{2} \phi}{\partial x^{2}}=\gamma_{e} n_{e}-\beta \gamma_{i} n_{i}+\delta \gamma_{b} n_{b}
\end{aligned}
$$


where $H=\sqrt{1+\xi^{2}}$ is the (dimensionless) enthalpy [52], wherein $\xi=\frac{h n_{e}}{4 m_{e} c}$; also, the $\gamma$ factor is rescaled as $\gamma_{j}=1 / \sqrt{1-\alpha u_{j}^{2}}$, where $\alpha=c_{s}^{2} / c^{2}=\mu_{e} \xi_{0}^{2}$ and $\xi_{0}=p_{F e} /\left(m_{e} c\right)=h n_{e 0} /\left(4 m_{e} c\right)$.

The time, space and dynamical variables have been rescaled as: $t \rightarrow \omega_{p i} t, x \rightarrow \omega_{p i} x / c_{s}, n_{j} \rightarrow n_{j} / n_{j 0}$, $u_{j} \rightarrow u_{j} / c_{s}$ (for $j=i, e, b$ ) and $\phi \rightarrow e \phi / 2 E_{F e}$, where we defined the ion plasma frequency $\omega_{p i}=\sqrt{Z_{i} e^{2} n_{e 0} / \epsilon_{0} m_{i}}$ (as in an $e-i$ plasma). We note that the ES potential scale $\left(2 E_{F e} / e\right)$ and the speed scale $c_{s}=\sqrt{2 Z_{i} E_{F e} / m_{i}}$ are functions of the (non-relativistic) electron Fermi energy $E_{F e}=p_{F e}^{2} / 2 m_{e}$ and the Fermi momentum $p_{F e}=$ $h n_{e 0} / 4$. The length scale is thus inferred as $c_{s} / \omega_{p i}$. A familiar reader will notice that $c_{s}$ is formally analogous to the acoustic (sound) speed in classical plasma modeling, although the underlying physics is clearly different, since thermal pressure is neglected in our model.

We retain, for latter reference, the definitions of: the ionto-electron charge ratio $\beta=\frac{Z_{i} n_{i 0}}{n_{e 0}}=1+\gamma_{b 0} \delta$, where $\gamma_{b 0}$ is redefined as $\gamma_{b 0}=1 / \sqrt{1-\alpha U_{b 0}^{2}}, U_{b 0}=\frac{u_{b 0}}{c_{s}}$ is the normalized equilibrium velocity of the beam; the beam ionto-electron number density ratio $\delta=\frac{Z_{b} n_{b 0}}{n_{e 0}}$; the electronto-ion mass ratio $\mu_{e}=\frac{m_{e}}{m_{i}}$ and, finally, the mass ratio $\mu_{b}=\frac{m_{b}}{m_{i}}$. Neutrality at equilibrium imposes $\beta=1+\gamma_{b 0} \delta$. The charge state for the positive ions to be $Z_{i}=1$ for simplicity. Low mass beam ions, viz. $\mu_{b} \sim 1$, will be assumed throughout. Furthermore, we assume that $\gamma_{b 0} \delta \ll 1$, in account of a low density beam, so as to avoid magnetization, in respect of the electrostatic approximation. It is emphasized that the latter implicit assumption is not explicitly adopted in the algebra, which remains general.

\section{TRAVELLING WAVE APPROXIMATION - PSEUDOPOTENTIAL FORMALISM}

Anticipating stationary profile excitations, we shall consider state perturbations propagating at (dimensionless) speed $V=\frac{U_{s o l}}{c_{s}}$ (implying a real physical speed $U_{s o l}$ ). Note, in passing, that $V$ is formally analogous to the so called "Mach number" in electrostatic soliton theory for classical plasmas $[4,5]$; that term (inspired by acoustics) is avoided here since, rigorously speaking, the "true" sound speed (and thus the "true" Mach number) ia actually configuration-dependent in multicomponent plasmas, hence this terminology may be misleading; see the rigorous discussion in [58].

Equations (9) above form a system of coupled nonlinear partial differential equations (PDEs), which is impossible to solve analytically. In order to get some insight, we shall consider a reference frame moving at the velocity $V$, by assuming that all quantities are functions of a single variable $X=x-V t$, viz.

$$
\frac{\partial}{\partial t}=-V \frac{\partial}{\partial X}, \quad \frac{\partial}{\partial x}=\frac{\partial}{\partial X} .
$$

Introducing this variable transformation in Eqs. (9), we obtain a system of (coupled) ordinary differential equations (ODEs):

$$
\begin{array}{lr}
-V\left(\gamma_{i} n_{i}\right)^{\prime}+\left(\gamma_{i} n_{i} u_{i}\right)^{\prime}=0, & \\
-V\left(\gamma_{e} n_{e}\right)^{\prime}+\left(\gamma_{e} n_{e} u_{e}\right)^{\prime}=0, & \\
-V\left(\gamma_{b} n_{b}\right)^{\prime}+\left(\gamma_{b} n_{b} u_{b}\right)^{\prime}=0, & \\
-V\left(\gamma_{i} n_{i}\right)^{\prime}+u_{i}\left(\gamma_{i} u_{i}\right)^{\prime}+\phi^{\prime}=0, & \\
-H\left(V-u_{e}\right)\left(\gamma_{e} u_{e}\right)^{\prime}+\frac{\gamma_{e} n_{e}}{H \mu_{e}}\left(1-\alpha V u_{e}\right) u_{e}^{\prime}-\frac{1}{\mu_{e}} \phi^{\prime}=0,
\end{array}
$$

coupled via Poisson's equation, now expressed as

$$
\phi^{\prime \prime}=\gamma_{e} n_{e}-\beta \gamma_{i} n_{i}+\delta \gamma_{b} n_{b}
$$

By taking into account suitable boundary conditions (namely, $\lim _{X \rightarrow \pm \infty} u_{b}=U_{b 0}$ for the ion beam, and vanishing conditions at infinity for the remaining variables), the latter equations provide the various fluid (state) variables in terms of the ES potential $\phi$. The long algebraic procedure is described in full detail in the Appendix. The above ODEs are thus disentangled into a single ODE for the electrostatic potential $\phi$, in the form:

$$
\frac{1}{2}\left(\frac{d \phi}{d X}\right)^{2}+S(\phi)=0 .
$$

The pseudopotential funstion $S$ is given by

$S(\phi)=\left(1+\gamma_{b 0} \delta\right) S_{i}(\phi)-\delta\left[S_{b 1}(\phi)-S_{b 0}\right]-\left[S_{e 1}(\phi)-S_{e 0}\right]$

where the terms emanating from the different plasma constituents to the latter expression (identified by the respective indices) are given by the expressions

$$
\begin{aligned}
S_{i}(\phi)= & V u_{i} \gamma_{i}, \quad S_{b 1}(\phi)=-\mu_{b} \gamma_{b 0} u_{b} \gamma_{b}\left(V-U_{b 0}\right), \\
S_{e 1}(\phi)= & {\left[\gamma_{e} n_{e}\left(\phi+\frac{H_{0}}{\xi_{0}^{2}}\right)\right.} \\
& \left.\quad-\frac{1}{2 \xi_{0}^{3}}\left(\sinh ^{-1}\left(\xi_{0} n_{e}\right)+\xi_{0} n_{e} H\right)\right]
\end{aligned}
$$

along with (their equilibrium counterparts)

$$
\begin{aligned}
S_{b 0} & =-\mu_{b} \gamma_{b 0}^{2} U_{b 0}\left(V-U_{b 0}\right), \\
\text { and } \quad S_{e 0} & =\frac{H_{0}}{\xi_{0}^{2}}-\frac{1}{2 \xi_{0}^{3}}\left(\sinh ^{-1}\left(\xi_{0}\right)+\xi_{0} H_{0}\right) .
\end{aligned}
$$

Note that $S_{j 1}(\phi=0)=S_{j 0}($ for $j=i, e, b)$, at equilibrium, while $S_{i 1}(\phi=0)=0$.

Eq. (17) is formally reminiscent of a pseudo-mechanical energy balance equation, characteristic of a conservative system. This (often called "Sagdeev") formalism [4] has been long known to describe localized electrostatic excitations (collisionless shocks) in plasmas [5].

At this stage, one is left with the task of solving Eq.(17) (numerically) for the electrostatic potential $\phi(X)$, and then calculating the remaining plasma variables (as functions of the travelling coordinate $X$ ), in the moving frame. 


\section{EXISTENCE REQUIREMENTS FOR ES SOLITARY WAVES}

Drawing inspiration from the classical theory [5], but also from the beam-free quantum model [52], it is expected that the soliton speed $V$ may take values in an interval, say, $\left(V_{1}, V_{2}\right)$, in order for real, physically acceptable solutions to exist. In classical models, the lower boundary $V_{1}$ corresponds to the "acoustic" speed (hence nonlinear waves will be superacoustic), while the upper limit $V$ physically corresponds to the infinite compression limit, where the density variable diverges [5]. Both limits, $V_{1}$ and $V_{2}$, depend on the particular configuration of the problem, i.e. in this case, the plasma composition and the intrinsic beam features. In the following, we shall determine the pulse speed limitations, and will then investigate the effect of the beam on the region of existence of electrostatic waves. Obviously, at every step, the (beamfree) limit $\delta=0$ agrees with the results in Ref. 52 for electron-ion (i.e., beam-free) plasma.

The form of the function $S$ should obey a number of constraints, to guarantee the reality of all physical variables involved. First of all, it is obvious that $S(\phi=$ $0)=\left.\frac{d S(\phi)}{d \phi}\right|_{\phi=0}=0$, since electric must vanish, thanks to neutrality, at equilibrium. The pseudopotential function possesses a maximum at the origin, viz. $\left.\frac{d^{2} S(\phi)}{d^{2} \phi}\right|_{\phi=0}<0$. Finally, we emphasize that the dynamics will be limited between equilibrium and $\phi_{0}$, say, denoting the first nonzero root of $S$, suggesting a maximum value $\phi_{0}$ for $\phi$.

a. The supersonic condition: $V \geq V_{1}$. The requirement $\frac{d^{2} S(\phi)}{d \phi^{2}} \leq 0$ (see above) implies:

$$
\begin{aligned}
\left(1+\gamma_{b 0} \delta\right) \frac{1}{V_{1}^{2}} & -\frac{H_{0}}{1-\mu_{e} H_{0}^{2} V_{1}^{2}} \\
& +\frac{\delta}{\mu_{b}} \frac{1-\alpha U_{b 0} \gamma_{b 0}^{2}\left(V_{1}-U_{b 0}\right)}{\gamma_{b 0}^{3}\left(V_{1}-U_{b 0}\right)^{2}} \leq 0 .
\end{aligned}
$$

The lower value for the soliton speed, say $V_{1}$, is therefore obtained upon solving the equation $S^{\prime \prime}\left(\phi=0 ; V_{1}\right)=0$ (numerically) for $V_{1}$.

The pulse speed threshold $V_{1}$ is depicted in Fig. 1: one notices that it is a growing function of $U_{b, 0}$ and $\delta$. In other words, a stronger beam current (i.e. a higher beam density or/and velocity values) leads to faster pulses, since slower ones will not occur and will not be observed. b. The upper speed limit (reality condition): $V<V_{2}$. An additional condition results from reality of the fluid state variables. The (reality of the) analytical expression for the (positive) ion fluid speed $u_{i}$ - see Eq. (A6) in the Appendix - leads to the analytical requirement:

$$
0<\phi \leq \phi_{\max ,+}=\frac{1}{\alpha}\left(1-\sqrt{1-\alpha V_{2}^{2}}\right) .
$$

This inequality is obviously satisfied for negative values of $\phi$, and thus needs to be considered as a constraint only for positive $\phi$, whose value will be limited by $\phi_{\max ,+}$ above. In the non-relativistic limit $\alpha \ll 1$, this condition reduces to $\phi \leq \phi_{\max ,+}=V_{2}^{2} / 2$, which is the well known

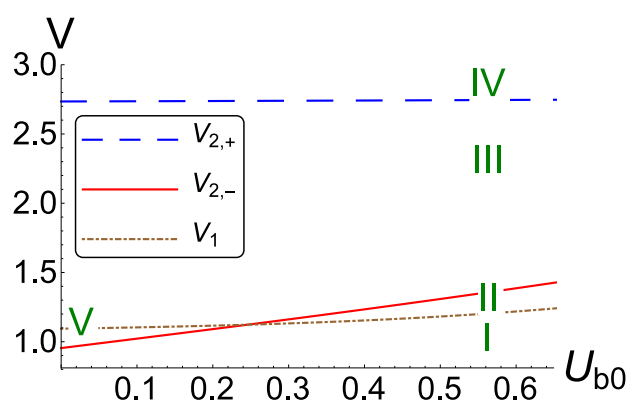

(a)

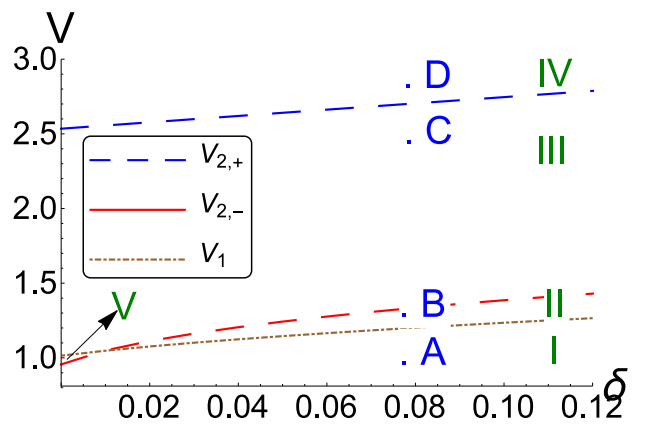

(b)

FIG. 1: (Color online) The existence regions (intervals of values) in terms of the normalized pulse speed (pseudo-"Mach number") $V$ are depicted, for various values of the electron density $n_{e 0}$, (a) versus the beam velocity $U_{b 0}$ (for $\delta=0.1$ ), and (b) versus the beam density $\delta$ (for $U_{b 0}=0.6$ ). In both plots, we have taken $\xi_{0} \simeq 0.06038$ (corresponding to $n_{e 0}=10^{11} \mathrm{~m}^{-1}$ ) and $\mu_{b}=1$. Note the existence of five (5) regions (labelled as I, II, III, IV and V, as discussed in Section IV). Four (4) representative points are identified in the bottom panel (tagged by letters A, B, C and D); see the discussion in Section V.

classical requirement $[4,5]$.

Imposing $S\left(\phi_{\max ,+}\right) \geq 0$ and solving the corresponding inequality numerically leads to the value(s) of the upper (pulse speed) limit $V_{2,+}$ for positive pulses; see in Fig. 1. Following a similar argument, from Eq. (A10) (in the Appendix) for the beam fluid speed, we find the following condition to be imposed, for reality:

$\phi_{\max ,-}=-\frac{\mu_{b}}{\alpha}\left[\gamma_{b 0}\left(1-\alpha V_{2} U_{b 0}\right)-\sqrt{1-\alpha V_{2}, 2}\right] \leq \phi \leq 0$,

i.e., in absolute value,

$0 \leq|\phi| \leq \frac{\mu_{b}}{\alpha}\left[\gamma_{b 0}\left(1-\alpha V_{2} U_{b 0}\right)-\sqrt{1-\alpha V_{2}^{2}}\right]=\left|\phi_{\max ,-}\right|$,

This inequality is obviously satisfied for positive values of $\phi$; it thus needs to be considered as a constraint only 


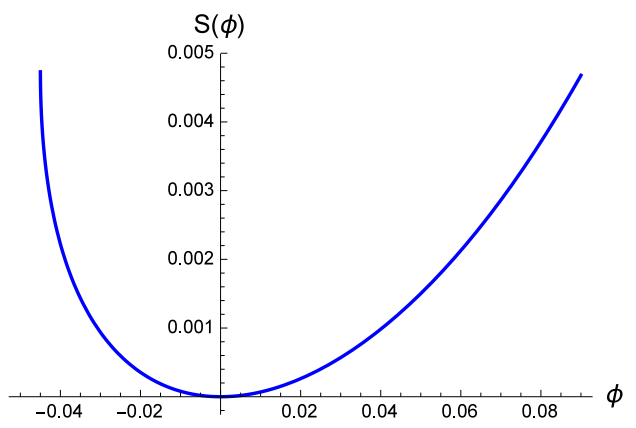

(a)

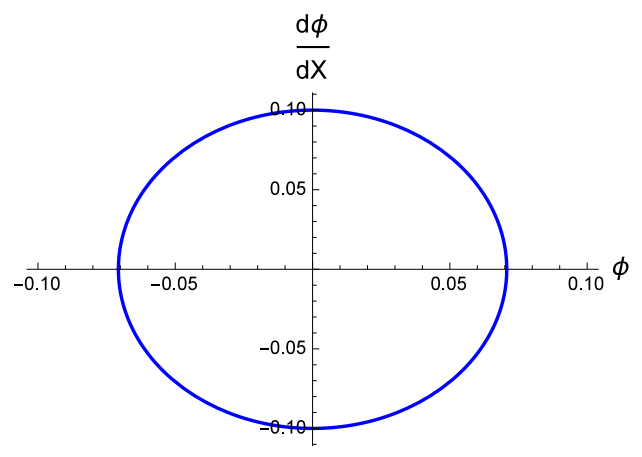

(b)

FIG. 2: (Color online) Point A in Fig. 1 is shown. (a) The pseudopotential $S$ and (b) the corresponding phase portrait are depicted, versus the electrostatic potential $\phi$. The parameter values are: $V=0.9, \delta=0.1, \xi_{0} \simeq 0.06038$ (or $n_{e 0}=10^{11} \mathrm{~m}^{-1}$ and $\mu_{b}=1$ and $U_{b 0}=0.6$. It is obvious that localized modes (pulses) do not exist in this plasma configuration.

for negative $\phi$, whose (absolute) value will be limited by $\left|\phi_{\text {max },-}\right|$ above, as obvious in (24). In the non-relativistic limit $\alpha \ll 1$, we obtain $\phi_{\max ,-}=-\frac{\mu_{b}}{2}\left(V_{2}-U_{b 0}\right)^{2}$, hence for $U_{b 0}=0$, we recover $\phi_{\max ,-}=-\frac{V_{2}^{2}}{2}$, again in agreement with the infinite compression limit in the classical case $[4,5]$.

Imposing $S\left(\phi_{\max ,-}\right) \geq 0$ and solving the corresponding inequality numerically yields the value(s) of the upper (pulse speed) limit $V_{2,-}$ for negative pulses to exist; notice the corresponding curve in Fig. 1.

We see that a maximum value must be imposed for electrostatic potential $(\phi)$ pulses to occur, whether positive or negative, viz. $V \leq \min \left\{V_{2,+}, V_{2,-}\right\} \equiv V_{\max }$. In particular, positive pulses will exist if $V \leq V_{2,+}-$ see Fig. 4 for an example - while negative pulses will exist if $V$ takes values in the interval $V \leq V_{2,-}$. As it turns out, both conditions may also be satisfied simultaneously; see e.g. Fig. 3 for an example. We have found no region of values where only negative pulses exist. Physically, this is due to the fact that the tenuous beam represents, by defini-

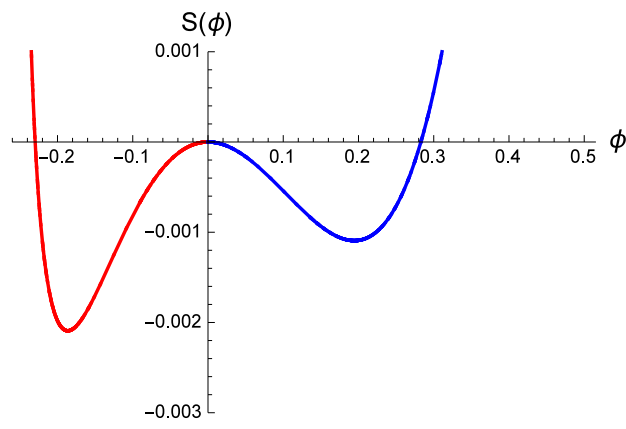

(a)

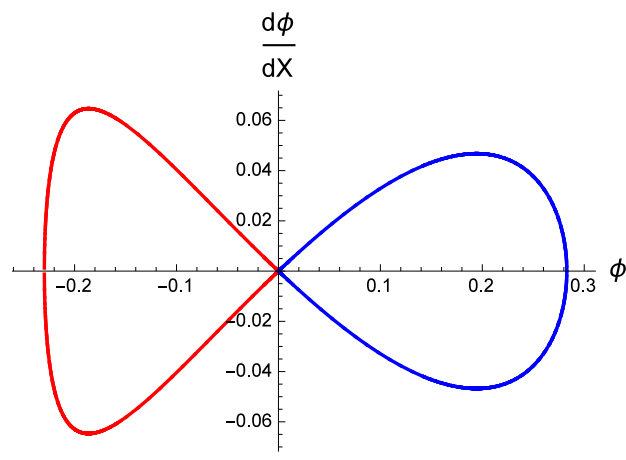

(b)

FIG. 3: (Color online) Point B in Fig. 1 is shown. (a) The pseudopotential $S$ and (b) the corresponding phase portrait are depicted, versus the electrostatic potential $\phi$. The parameter values are: $V=1.3, \delta=0.1, \xi_{0} \simeq 0.06038$ (or $n_{e 0}=10^{11} \mathrm{~m}^{-1}$ and $\mu_{b}=1$ and $U_{b 0}=0.6$. Both positive and negative potential pulses may exist in this plasma configuration, as predicted in Fig. 1.

tion, a minority ion population, i.e. is not the dominant inertial species in the plasma mixture considered.

In the above considerations, it is understood that $V_{2}<$ $1 / \sqrt{\alpha}$, a condition which holds for all realistic parameter values considered.

Combining the above reality requirement(s), we henceforth impose the condition: $S\left(\phi=\phi_{m}\right) \geq 0$. The upper boundary $V_{2}$ is thus obtained by solving the equation $S\left(\phi=\phi_{m} ; V_{2}\right)=0$ (numerically) for $V_{2}$.

The range of permitted values (for pulse existence), viz. $V \in\left[V_{1}, V_{2, \pm}\right]$, was determined numerically, as described above. It is shown in Fig. 1, against the electron density $U_{e b 0}$ (for given fixed values of $\delta$ ) and, reversely, versus the beam-to-electron density ratio $\delta$ (for given fixed values of $\left.U_{b 0}\right)$. One distinguishes five (5) regions in Fig. 1:

- Region I: $V<V_{1}\left(<V_{2, \pm}\right)$;

- Region II: $V_{1}<V<V_{2,-}<V_{2,+}$;

- Region III: $V_{1}<V_{2,-}<V<V_{2,+}$; 


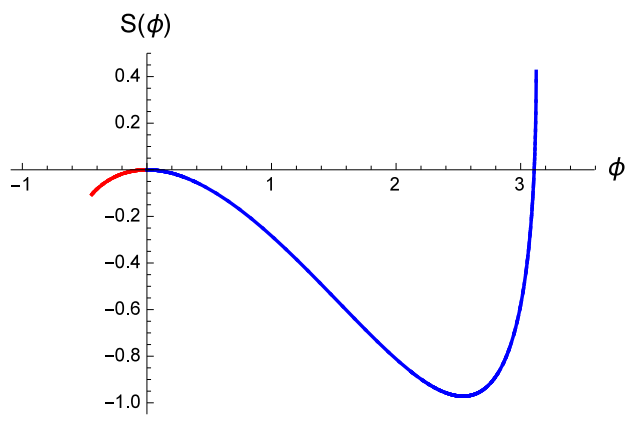

(a)

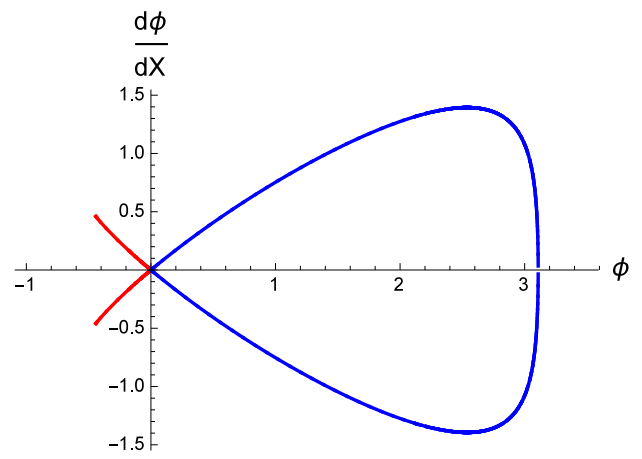

(b)

FIG. 4: (Color online) Point C in Fig. 1 is shown. (a) The pseudopotential $S$ and (b) the corresponding phase portrait are depicted, versus the electrostatic potential $\phi$. The parameter values are: $V=2.5, \delta=0.1, \xi_{0} \simeq 0.06038$ (or $n_{e 0}=10^{11} \mathrm{~m}^{-1}$ and $\mu_{b}=1$ and $U_{b 0}=0.6$. Only positive potential pulses may exist in this plasma configuration, as predicted in Fig. 1.

- Region IV: $V>\max \left\{V_{2,+}, V_{2,-}\right\}$;

- Region $\mathrm{V}$ : $V_{2,-}<V<V_{1}<V_{2,+}$.

For illustration purposes, we have identified four (4) points (tagged by letters A, B, C and D in Fig. 1b), representative of configurations in Regions I, II, III and $\mathrm{IV}$, respectively. (Region $\mathrm{V}$ is formally equivalent to $\mathrm{I}$, since no pulses will occur therein.)

For $V<V_{1}$ (region I), the pseudopotential $S(\phi)$ possesses no minimum; the system admits no localized solitary solutions, see Fig. 2.

For $V_{1}<V<V_{2,-}<V_{2,+}$ (region II), the pseudopotential function has two wells, one on the positive side and the other on the negative side of the $\phi$ axis, as shown in Fig. 3. Hence, either positive or negative localised solitary pulses may exist.

In region III, where $V_{1}<V_{2,-}<V<V_{2,+}$, as shown in Fig. 4, only positive localized solitary pulses may occur, since a potential well (a minimum) occur only on the positive semiaxis.

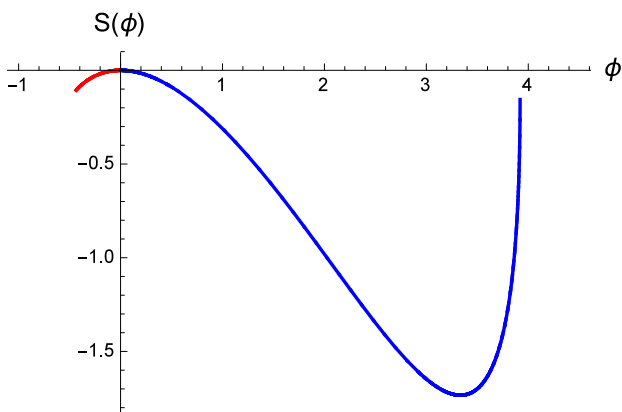

(a)



(b)

FIG. 5: (Color online) Point D in Fig. 1 is shown. (a) The pseudopotential $S$ and (b) the corresponding phase portrait are depicted, versus the electrostatic potential $\phi$. The parameter values are: $V=2.8, \delta=0.1, \xi_{0} \simeq 0.06038$ (or $n_{e 0}=10^{11} \mathrm{~m}^{-1}$ and $\mu_{b}=1$ and $U_{b 0}=0.6$. No localized modes (pulses) exist in this plasma configuration, as evident in Fig. 1, since the free state variable $\phi$ ceases to be real before a root of $S$ occurs.

Finally, for a large pulse speed $V>V_{2,+}$ (region IV), we see from Fig. 5 that localised solitary pulses do not exist. As said above, region $\mathrm{V}$ is amenable to the case of region I, since $V<V_{1}$, hence no localized solutions exist.

\section{PARAMETRIC INVESTIGATION}

The system of differential equations (17) has been solved numerically, for various values of the plasma configurational parameters $n_{e 0}, U_{b 0}, \delta$ and $V$ (for a given value of $\left.\mu_{b}\right)$. The results are depicted in Figs. 6-13. We have investigated how plasma configurational parameters may affect parametrically the ES potential pulse (form), the corresponding bipolar electric field $E$ and the various plasma state (fluid density, speed) variables. Our main conclusions are summarized in this Section.

c. Negative ion beam speed. The influence of the initial beam velocity of the negative ions on the shape of 


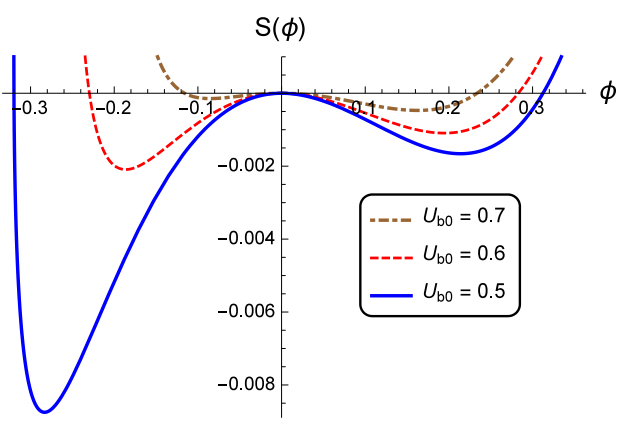

(a)



(b)

FIG. 6: (Color online) (a) The pseudopotential $S(\phi)$ versus $\phi$, and (b) the corresponding phase portrait $(\phi, d \phi / d X)$ are depicted, for various values of the beam velocity $U_{b 0}$. The values of the parameters are $V=1.3, \delta=0.1, n_{e 0}=10^{11} \mathrm{~m}^{-1}\left(\right.$ or $\left.\xi_{0} \simeq 0.06038\right)$ and $\mu_{b}=1$.

the pseudopotential (function) $S$ and the corresponding phase portrait is depicted in Fig. 6, for a representative set of values from region II, where coexistence (of positive and negative pulses) is manifested. From Fig. 6a, we can see that the pseudopotential (function) $S$ is characterized by two wells, one for positive and one for negative values of the electrostatic potential $\phi$. Interestingly, the roots are of similar order of magnitude (in absolute value) for, e.g., $U_{b 0}=0.5$ (see the blue, continuous curves in Fig. 6a) resulting to pulses of similar strength, but of opposite polarity in the ES potential (as shown in Fig. 7a). However, the different form of the pseudopotential (steeper and deeper in the left, in Fig. 6a) leads to distinct Efield forms, as depicted in Fig. 6b (compare the blue, continuous curves there).

We note that, by increasing the beam velocity, both the amplitude and the depth of both pseudopotential wells decreases. We also notice that the negative potential has larger amplitude and is deeper than the positive potential for the same beam velocity value, thus leading to sharper, narrower and steeper excitations in the electric field; see Fig.7a. From the topology of the phase portrait, in Fig. 6b, one can see two heteroclinic orbits on either side of the origin $(0,0)$, a positive and a negative one, which correspond respectively to two types of solitary wave solution. The state variables of the different species are depicted for different values of the beam velocity in Fig.7. Figs.7(a, b) show the electrostatic potential $\phi$ and the corresponding electric field $E$. We can see that there is coexistence of positive and negative structures in both the electrostatic potential and the electric field. The density and the flow velocity variables of the electrons, $n_{e}(X)$ and $u_{e}(X)$, are shown in Figs.7(c, d), while the corresponding plots for the ion fluid are shown in Figs.7(e,f); similarly, the number density and the flow velocity $n_{b}(X), u_{b}(X)$ for the beam fluid are shown in Figs7 $(\mathrm{g}, \mathrm{h})$.
It is interesting to point out that a positive potential pulse (disturbance) may be simultaneously associated with a positive density variation (compression) in the positive ion component, but with a negative density variation (rarefaction) in the beam ion component, cf. e.g. Figs.7 (e,g). The same is true of negative potential pulses. For the sake of rigor, we have therefore had to abandon the common terminology (referring to "compressive" or "rarefactive" solitons), which we find rather misleading. Instead, we will refer to positive potential pulses (PPP) and negative potential pulses (NPP), two expressions whose meaning is clear; see. Fig.7. In order to distinguish the (sets of) curves resulting from PPP or NPP, respectively, we have labeled all graphs in Fig.7 accordingly.

For the sake of clarity, and for direct comparison with the above (for region II), we have also elaborated the analogous plots for representative parameter values from Region III, where only positive localized structures may occur - recall Fig. 1 - as indeed observed in Fig. 8. The corresponding state variables are depicted in Fig. 9. As obvious from Fig. 9(a, b), the electrostatic potential (excitation) is manifested as a positive pulse, giving rise to an bipolar electric field structure of the usually expected polarity, for ion acoustic waves (only). The ions and the electrons are characterized by a localized density increase (compression) while, on the other hand, the beam suffers a density decrease (a rarefaction), as evident in Fig. 9.

d. Negative ion beam density effect. The effect of the beam density (via the ratio/parameter $\delta$ ) on the shape of the pseudopotential $S(\phi)$ and on the topology of the phase portrait $\left(\frac{d \phi}{d X}, \phi\right)$ is shown in Fig. 10, again starting with typical values from region II ( $\mathrm{PPP} / \mathrm{NPP}$ coexistence). As seen in Fig. 10, the width and the depth of the potential well of the pseudopotential decreases (in absolute value) with a an increase in (the beam density) $\delta$. The electrostatic potential $(\phi)$ and electric field $(E)$ 


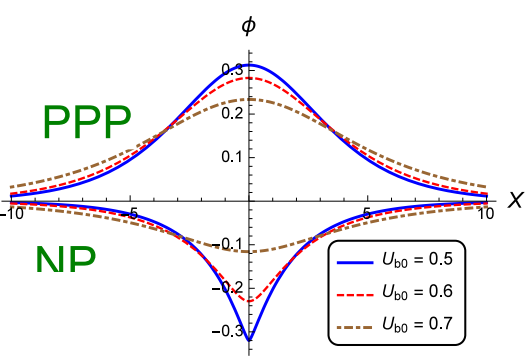

(a)

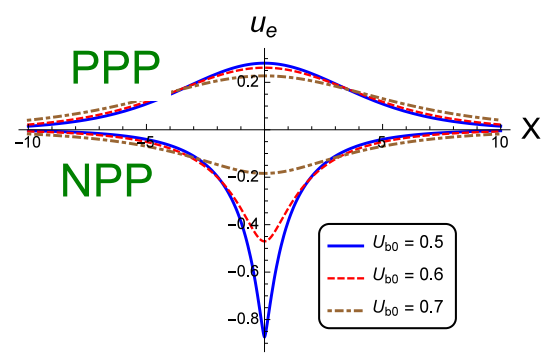

(d)

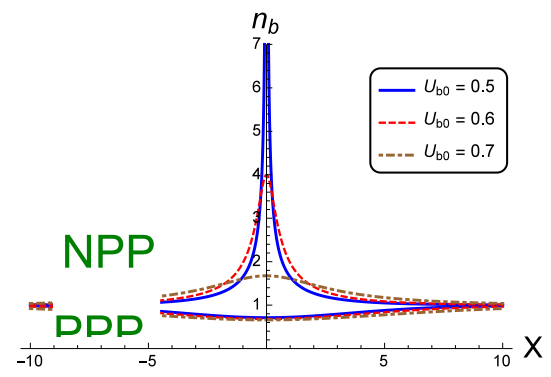

$(\mathrm{g})$

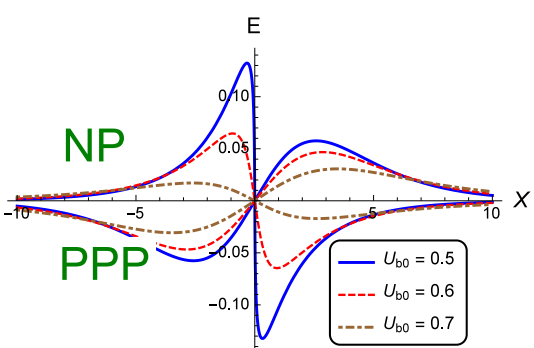

(b)

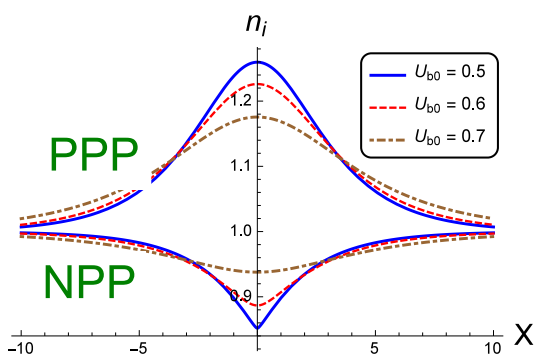

(e)

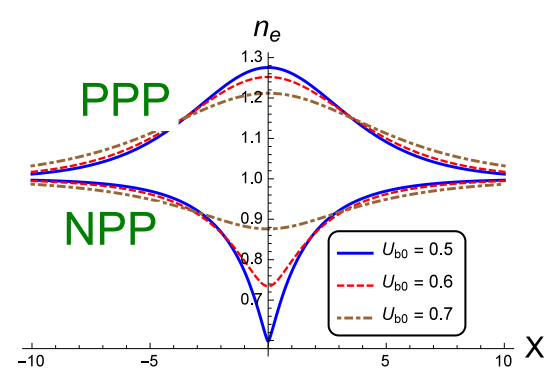

(c)

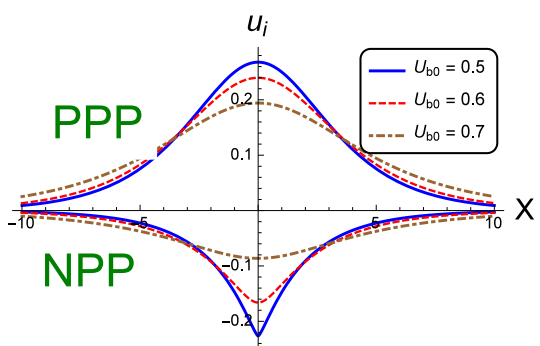

(f)

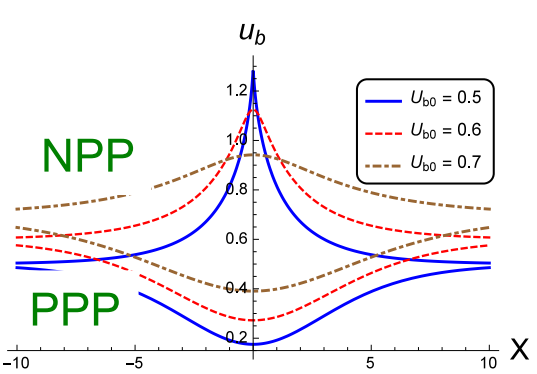

(h)

FIG. 7: (Color online) The plasma (fluid) state variables are shown in terms of the space variable $X$, for different values of beam velocity $U_{b 0}$. We have taken $V=1.3, n_{e 0}=10^{11} \mathrm{~m}^{-1}, \delta=0.1$ and $\mu_{b}=1$.

disturbances, as well as the associated localized plasma state variable variations, are depicted for (different beam density values) in Fig. 11.

For the sake of comparison, we have plotted the same quantities as in Figs. 10 and 11 (varying $\delta$ ), but for a value of $V$ representative of region III, where only positive potential excitations occur, as discussed above. The results are shown in Figs. 12 and13, where the influence of the beam density (via $\delta$ ) is obvious. We see in Fig.13(a, b) that only PPP structures exist in this case, while the (positive) ion and the electron fluids are compressed and accelerated, whereas the (negative) ion beam fluid is rarefied and slowed down, as shown in Figs.13(g, h). The amplitude of these ES potential pulses decreases as the beam density (value) increases: see Figs.13(c - f).

\section{CONCLUSIONS}

By using a quantum hydrodynamic model, a theoretical model for the interaction of a tenuous negative-ion beam with an ultradense electron-ion plasma has been developed, where all plasma species were treated as inertial fluids. Based on a pseudopotential (so called, Sagdeev type) method, the effect of the beam on the geometric properties (shape) and on the propagation characteristics (velocity range) of localized electrostatic potential excitations (relativistic pulses) has been investigated. Five dif- 


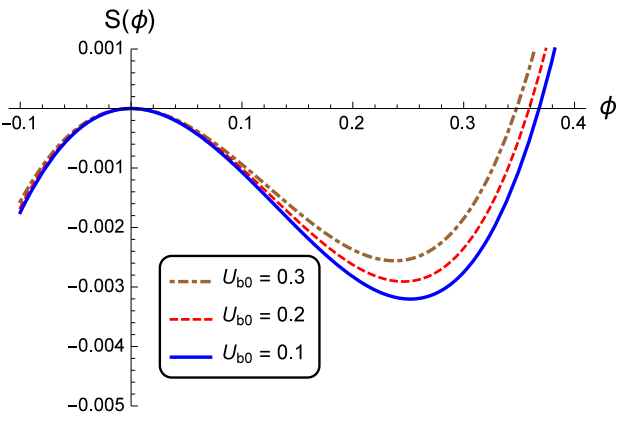

(a)



(b)

FIG. 8: (Color online) (a) The pseudopotential $S(\phi)$ and (b) the corresponding phase portrait are depicted, for different values of $U_{b 0}$. The parameter values adopted here are $V=1.3, \delta=0.1, n_{e 0}=10^{11} \mathrm{~m}^{-1}$ (or $\left.\xi_{0} \simeq 0.06038\right)$ and $\mu_{b}=1$.

ferent regions have been identified, in terms of the pulse speed $V$, characterized by different dynamical and structural characteristics of the electrostatic pulses. Importantly, in a certain region, both polarities of electrostatic pulses may exist, even simultaneously. The solution's characteristics (shape, topology) depends sensitively on the beam characteristics.

It may be mentioned, for rigor, that our analysis is reminiscent of the procedure adopted in Ref. 54, where a positively charged ion beam was considered. Although the algebraic model therein is formally analogous - though not identical - to our model here, the results are clearly distinct, as one might have expected from the outset (since negatively charged ions obviously affect the charge and current balance in a non-trivial, and often counterintuitive way). We have here shown that considering a negative ion beam opens a wide window into new possibilities in the dynamics, as it allows for co-existence of positive and negative pulses: the negative beam density and velocity may thus be considered as an order parameter, in some sense, allowing for significant alterations in (and enabling control and tuning of the characteristics of) localized modes occurring in the plasma.

Our theoretical investigation aimed at elucidating the dynamical behavior of localized electrostatic potential pulses in quantum relativistic plasmas. We have elaborated, in particular, a set of analytical conditions for the coexistence of positive and negative potential pulses. Our findings may be of relevance in astrophysical environments and in high-density laser-matter interaction experiments (both current and future), where ultrahigh density plasma configurations may form.

We have shown that the interaction of an ion beam with a quantum state (degenerate) plasma can favor the excitation of electrostatic solitary waves, which can be a mechanism for acceleration of electrons to ultrahigh energies. This picture is in agreement with earlier results, based on a Hall-MHD model and, independently, on rel- ativistic electromagnetic PIC simulations [46, 48].

\section{Acknowledgments}

The authors acknowledge support from the EU-FP7 IRSES Programme (grant 612506 QUANTUM PLASMAS FP7-PEOPLE-2013-IRSES). We warmly acknowledge a series of inspiring interactions with Dr Fernando Haas, during our respective visits to Instituto de Física, Universidade Federal do Rio Grande do Sul (Porto Alegre, Brazil). The authors express their gratitude for the hospitality provided by the Institute of Theoretical Physics (IFT) at the State University of Sao Paulo (UNESP - Universidade Estadual Paulista), and Professor Roberto Kraenkel in particular, where the latter stages of this work were carried out. IK is grateful for support from the Foundation for Research Support of the State of São Paulo (FAPESP), in the form of a Visiting Researcher fellowship, during the same period. Finally, one of us (ISE) acknowledges financial support via an Egyptian government fellowship.

\section{APPENDIX A: DERIVATION OF EXPRESSIONS FOR THE STATE VARIABLES IN THE MOVING FRAME}

The continuity Eqs. (10), (11) and (12) - in the moving frame - for the ion, electron and the beam-ion density can be integrated, respectively, leading to

$$
\begin{aligned}
& n_{i}=\frac{V}{\gamma_{i}\left(V-u_{i}\right)}, \\
& n_{e}=\frac{V}{\gamma_{e}\left(V-u_{e}\right)}, \\
& n_{b}=\frac{\gamma_{b 0}\left(V-U_{b 0}\right)}{\gamma_{b}\left(V-u_{b}\right)},
\end{aligned}
$$




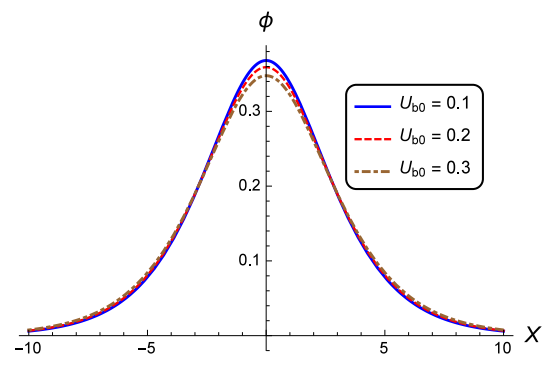

(a)

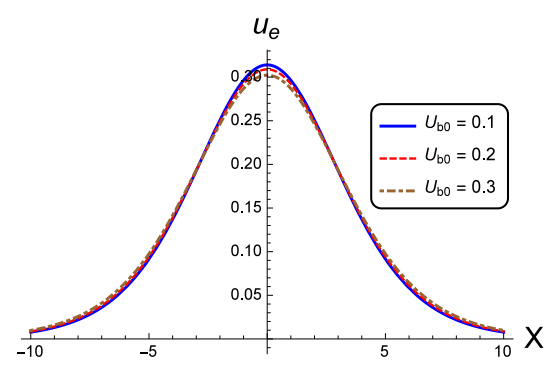

(d)

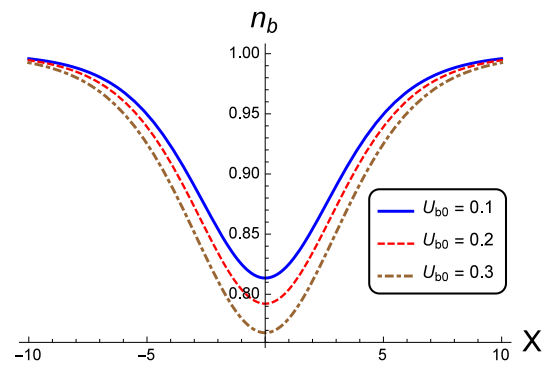

$(\mathrm{g})$

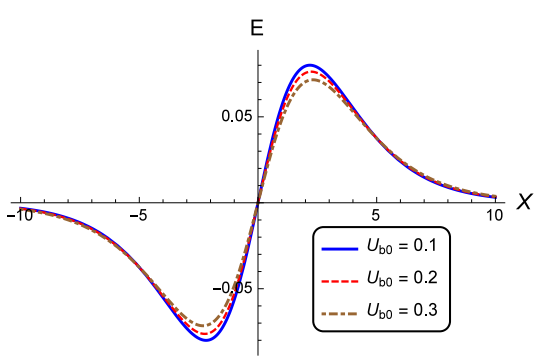

(b)

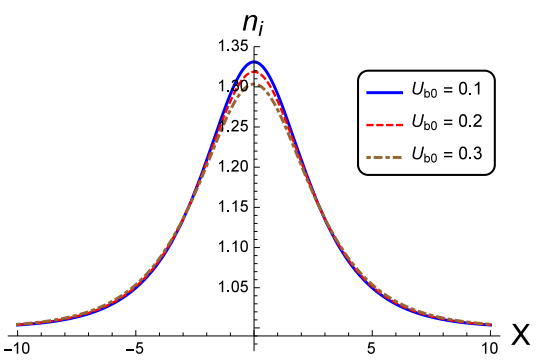

(e)

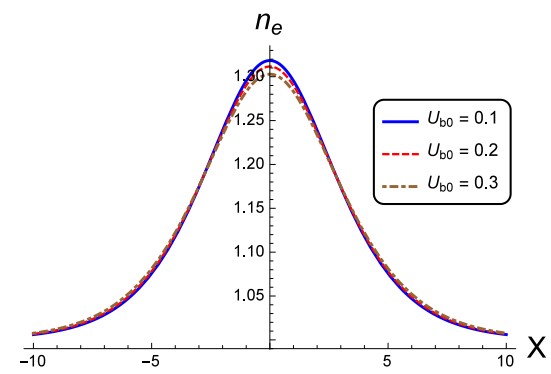

(c)

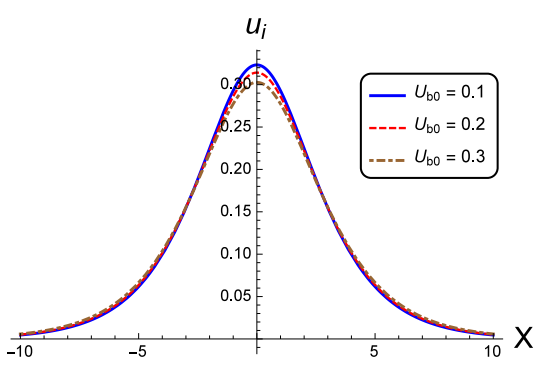

(f)

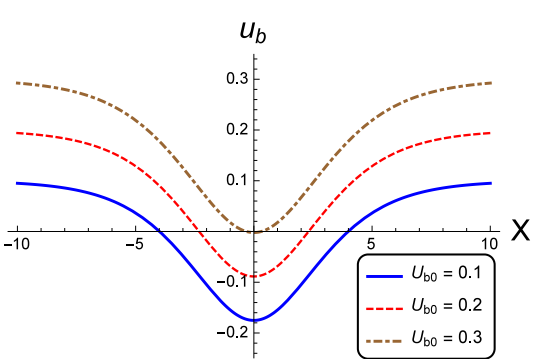

(h)

FIG. 9: (Color online) The plasma (fluid) state variables are shown in terms of the space variable $X$, for different values of beam velocity $U_{b 0}$. We have taken $V=1.3, n_{e 0}=10^{11} \mathrm{~m}^{-1}, \delta=0.1$ and $\mu_{b}=1$.

where $\gamma_{b 0}=1 / \sqrt{1-\alpha U_{b 0}^{2}}$ and specific boundary values at infinity were assumed, namely: $\lim _{X \rightarrow \pm \infty} n_{i, e, b}=$ $1, \lim _{X \rightarrow \pm \infty} u_{i, e}=0, \quad \lim _{X \rightarrow \pm \infty} u_{b}=U_{b 0}$ and $\lim _{X \rightarrow \pm \infty} \phi=0$.. In a similar way, the equation of motion (13) for the ions, we obtain

i.e.

$$
\phi=V \gamma_{i} u_{i}-\frac{\gamma_{i}}{\alpha}+\frac{1}{\alpha} .
$$

$$
\left[V^{2}+\alpha\left(\frac{1}{\alpha}-\phi\right)^{2}\right] u_{i}^{2}-\frac{2 V}{\alpha} u_{i}+\left[\frac{1}{\alpha^{2}}-\left(\frac{1}{\alpha}-\phi\right)^{2}\right]=0 .
$$

The solution for the positive ion-fluid speed reads:

$$
u_{i}=\frac{\frac{V}{\alpha}-\sqrt{\frac{V^{2}}{\alpha^{2}}-\left[V^{2}+\alpha\left(\frac{1}{\alpha}-\phi\right)^{2}\right]\left[\frac{1}{\alpha^{2}}-\left(\frac{1}{\alpha}-\phi\right)^{2}\right]}}{V^{2}+\alpha\left(\frac{1}{\alpha}-\phi\right)^{2}},
$$

where we chose to proceed with the solution satisfying the boundary condition $\lim _{X \rightarrow \pm \infty} \phi=\lim _{X \rightarrow \pm \infty} u_{i}=0$. Reality of the positive ion fluid speed (A6) imposes

$$
\phi \leq \frac{1}{\alpha}\left(1-\sqrt{1-\alpha V^{2}}\right) .
$$

In an similar way, integrating equation (15) for the negative ion (beam) species, we have:

$$
-\frac{1}{\mu_{b}} \phi=V\left(\gamma_{b} u_{b}-\gamma_{b 0} U_{b 0}\right)-\frac{\gamma_{b}}{\alpha}+\frac{\gamma_{b 0}}{\alpha}
$$




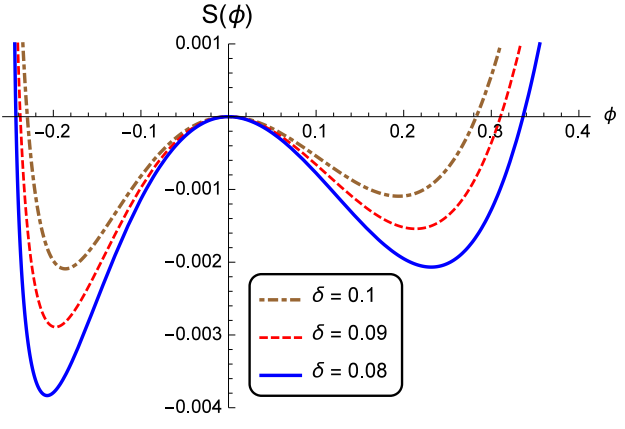

(a)

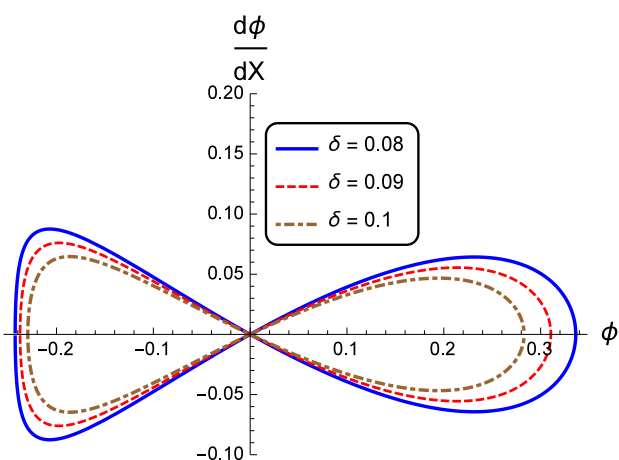

(b)

FIG. 10: (Color online) (a) The pseudopotential $S$ and (b) The corresponding phase portrait versus $\phi$ for different values of the beam-to-electron number density $\delta$. The values of the parameters are $V=1.3, U_{b 0}=0.6, n_{e 0}=10^{11} \mathrm{~m}^{-1}\left(\right.$ or $\left.\xi_{0} \simeq 0.06038\right)$ and $\mu_{b}=1$.

i.e.

$$
\left[V^{2}+\alpha\left[\gamma_{b 0}\left(V U_{b 0}-\frac{1}{\alpha}\right)-\frac{1}{\mu_{b}} \phi\right]^{2}\right] u_{b}^{2}-\frac{2 V}{\alpha} u_{b}+\left[\frac{1}{\alpha^{2}}-\left[\gamma_{b 0}\left(V U_{b 0}-\frac{1}{\alpha}\right)-\frac{1}{\mu_{b}} \phi\right]^{2}\right]=0 .
$$

Solving, we obtain

$$
u_{b}=\frac{\frac{V}{\alpha}-\sqrt{\left.\frac{V^{2}}{\alpha^{2}}-\left\{V^{2}+\alpha\left[\gamma_{b 0}\left(V U_{b 0}-\frac{1}{\alpha}\right)-\frac{1}{\mu_{b}} \phi\right]^{2}\right\}\left\{\frac{1}{\alpha^{2}}-\left[\gamma_{b 0}\left(V U_{b 0}-\frac{1}{\alpha}\right)-\frac{1}{\mu_{b}} \phi\right)\right]^{2}\right\}}}{V^{2}+\alpha\left[\gamma_{b 0}\left(V U_{b 0}-\frac{1}{\alpha}\right)-\frac{1}{\mu_{b}} \phi\right]^{2}},
$$

which satisfies: $\lim _{X \rightarrow \pm \infty} \phi=0$ and $\lim _{X \rightarrow \pm \infty} u_{b}=U_{b 0}$, as anticipated. A necessary condition for the beam velocity $u_{b}$ to be real-valued is:

$$
\phi \geq \phi_{\max , b}=-\frac{\mu_{b}}{\alpha}\left[\gamma_{b 0}\left(1-V U_{b 0} \alpha\right)-\sqrt{1-V^{2} \alpha}\right],
$$

must hold.

The positive ion density $n_{i}(\phi)$ and speed $u_{i}(\phi)$ are obtained, in terms of $\phi$ (for a given value of $V$ ), by combining Eqs. (A1) and (A6). The negative (beam) ion species state variables are obtained in an similar way, from (A3) and (A10).
Finally, equation (14) the electrons may now be integrated, to yield:

$$
\frac{\mu_{e}}{\alpha} \phi=\left[\gamma_{e} \sqrt{1+\frac{\alpha}{\mu_{e}} n_{e}^{2}}\left(1-\alpha V u_{e}\right)-H_{0}\right]
$$

or,

$$
\begin{aligned}
& {\left[\xi_{0}^{2}\left(1-\alpha V^{2}\right)\right] n_{e}^{4}} \\
& +\left[1-\left(\xi_{0}^{2} \phi+H_{0}\right)^{2}-\alpha V^{2}\left(1-\xi_{0}^{2}\right)\right] n_{e}^{2}+\alpha V^{2}=0 .
\end{aligned}
$$

The electron density is thus given, in terms of $\phi$, by the bi-quadratic polynomial equation

$$
n_{e}(\phi)=\sqrt{\frac{-\left[1-\left(\xi_{0}^{2} \phi+H_{0}\right)^{2}-\alpha V^{2}\left(1-\xi_{0}^{2}\right)\right]+\sqrt{\left[1-\left(\xi_{0}^{2} \phi+H_{0}\right)^{2}-\alpha V^{2}\left(1-\xi_{0}^{2}\right)\right]^{2}-4 \xi_{0}^{2}\left(1-\alpha V^{2}\right) \alpha V^{2}}}{2 \xi_{0}^{2}\left(1-\alpha V^{2}\right)}}
$$

Finally, in order to obtain the electron fluid speed in 


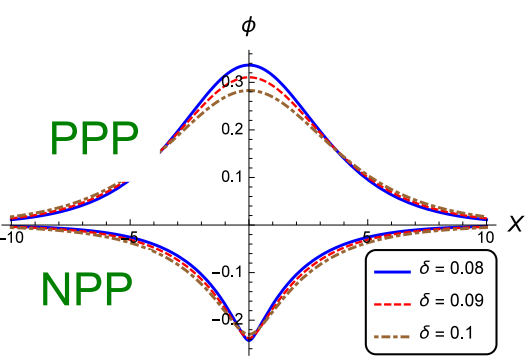

(a)

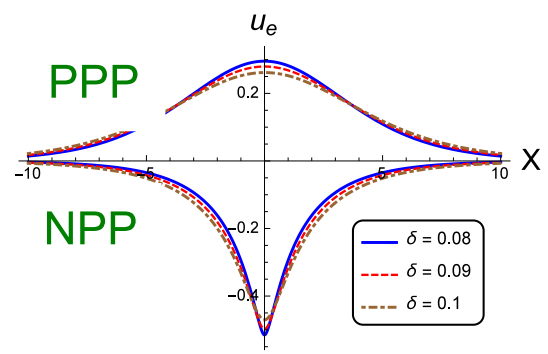

(d)

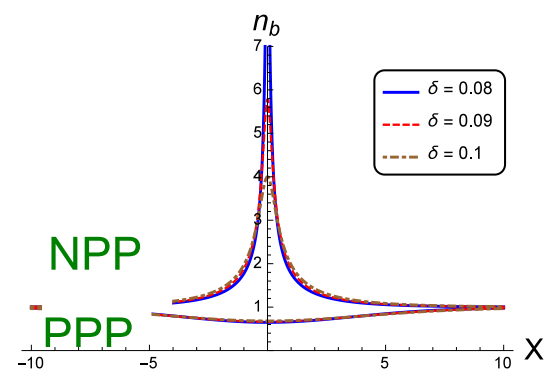

$(\mathrm{g})$



(b)

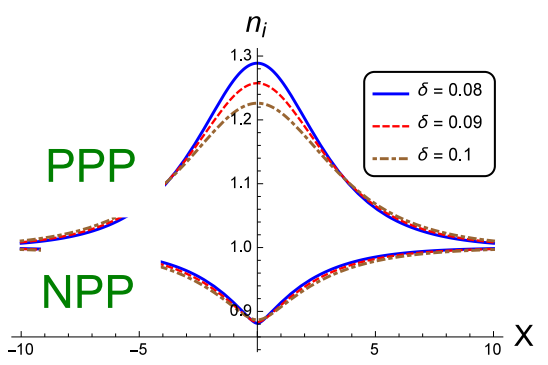

(e)



(c)

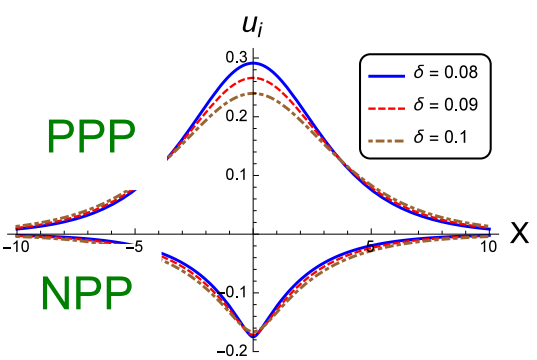

(f)

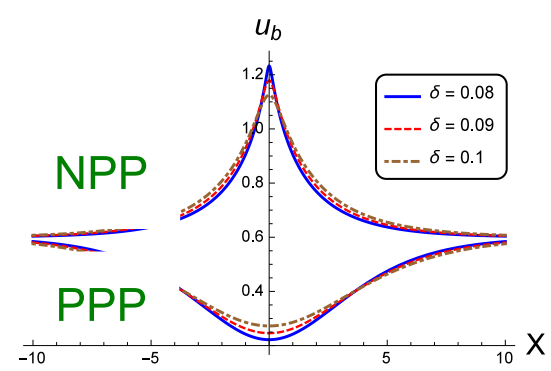

(h)

FIG. 11: (Color online) The plasma (fluid) state variables are shown in terms of the space variable $X$, for different values of the beam-to-electron number density $\delta$. We have taken $V=1.3, n_{e 0}=10^{11} \mathrm{~m}^{-1}, U_{b 0}=0.6$ and $\mu_{b}=1$.

Combining the above relations for the density variables into Poisson's equation (16), we find, for the electrostatic potential $\phi$, a differential equation in the form:

$$
\frac{d^{2} \phi}{d X^{2}}=f(\phi)
$$

where the function in the RHS is given by: 


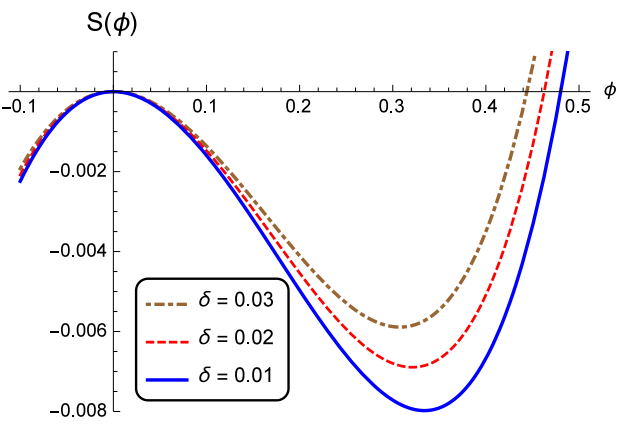

(a)

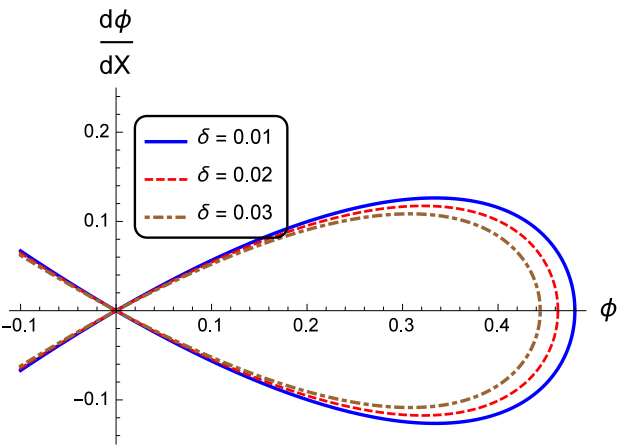

(b)

FIG. 12: (Color online) (a) The pseudopotential $S(\phi)$ and (b) the corresponding phase portrait are depicted for different values of the beam-to-electron number density $\delta$. The values of the parameters adopted in these plots are $V=1.3, U_{b 0}=0.6$, $n_{e 0}=10^{11} m^{-1}$ (or $\left.\xi_{0}=0.06038\right)$ and $\mu_{b}=1$.

$$
\begin{aligned}
f(\phi)= & -\frac{\alpha V^{2}}{1-\alpha V^{2}} \\
& +\frac{\sqrt{\alpha V^{2}+\frac{\mu_{e}}{2 \alpha}\left\{\sqrt{\left[1-\left(\xi_{0}^{2} \phi+H_{0}\right)^{2}-\alpha V^{2}\left(1-\xi_{0}^{2}\right)\right]^{2}-4 \xi_{0}^{2}\left(1-\alpha V^{2}\right) \alpha V^{2}}-\left[1-\left(\xi_{0}^{2} \phi+H_{0}\right)^{2}-\alpha V^{2}\left(1-\xi_{0}^{2}\right)\right]\right\}}}{1-\alpha V^{2}} \\
& -\left(1+\gamma_{b 0} \delta\right) \frac{V}{V-\frac{\frac{V}{\alpha}-\sqrt{\frac{V^{2}}{\alpha^{2}}-\left[V^{2}+\alpha\left(\frac{1}{\alpha}-\phi\right)^{2}\right]\left[\frac{1}{\alpha^{2}}-\left(\frac{1}{\alpha}-\phi\right)^{2}\right]}}{V^{2}+\alpha\left(\frac{1}{\alpha}-\phi\right)^{2}}} \\
+ & \delta \frac{\gamma_{b 0}\left(V-U_{b 0}\right)}{V^{2}}
\end{aligned}
$$

Multiplying by the derivative $d \phi / d X$ and integrating, we obtain precisely Eq. (17), viz. $\frac{1}{2}\left(\frac{d \phi}{d X}\right)^{2}+S(\phi)=0$.

where $S$ is a nonlinear function given by Eq. (18).

[1] L. Romagnani, S. V. Bulanov, M. Borghesi, P. Audebert, J. C. Gauthier, K. Lwenbrck, A. J. Mackinnon, P. Patel, G. Pretzler, T. Toncian, and O. Willi, Phys. Rev. Lett. 101, 025004 (2008).

[2] H. Ahmed, M.E. Dieckmann, L. Romagnani, Domenico Doria, Gianluca Sarri, M. Cerchez, E. Ianni, I. Kourakis, A. L. Giesecke, M. Notley, R. Prasad, K. Quinn, O. Willi, M. Borghesi, Phys. Rev. Lett., 110, 205001 (2013).

[3] R. Pottelette, R. A. Treumann, M. Berthomier, J. Jasperse, Nonlin. Proc. Geophys. 10, 87 (2003); J. S. Pickett, L.-J. Chen, S. W. Kahler, O. Santolik, D. A. Gurnett, B. T. Tsurutani, and A. Balogh, Annales Geophysicae 22, 2515 (2004).

[4] R. Z. Sagdeev, Cooperative phenomena and shock waves in collisionless plasmas, Rev. Plasma Phys., Vol. 4, M. A. Leontovich, Ed. New York: Consultants Bureau (1966), p. 52.

[5] F. Verheest and M.A. Hellberg, Electrostatic Solitons and Sagdeev Pseudopotentials in Space Plasmas: Review of Recent Advances, in Handbook of Solitons (S.P. Land and S.H. Bedore, Eds.), Nova Science Publ. (2009).

[6] F. Verheest, M.A. Hellberg and I. Kourakis, Phys. Rev. E, 87 (4), 043107 (2013).

[7] B. Song, N. DAngelo, and R. L. Merlino, Phys. Fluids B 3, 284 (1991).

[8] N. Sato, Plasma Sources Sci. Technol. 3, 395 (1994).

[9] K. Koga and Y. Kawai, Jpn. J. Appl. Phys., Part 1 38, 1553 (1999). 


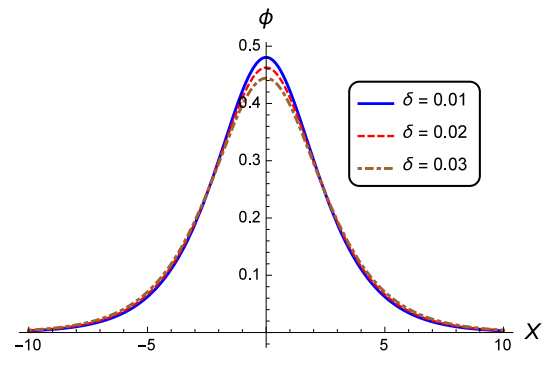

(a)

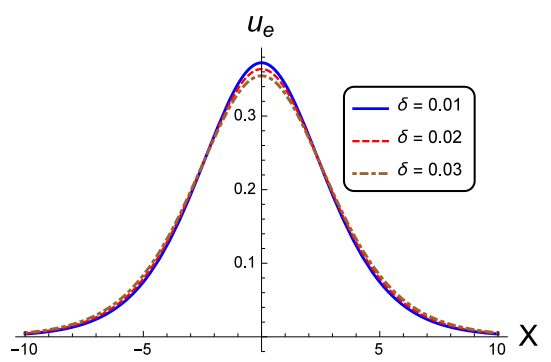

(d)

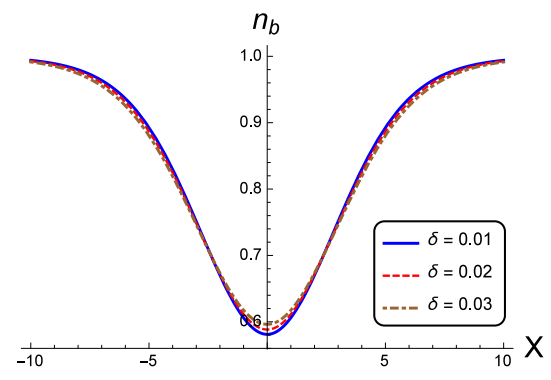

$(\mathrm{g})$

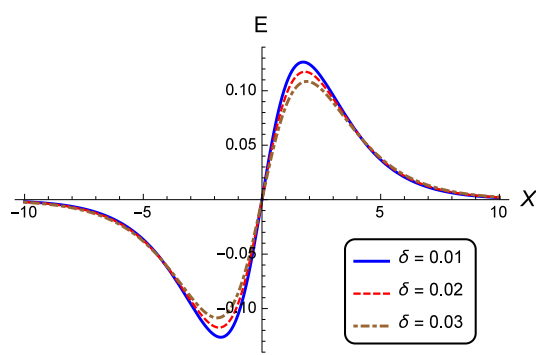

(b)

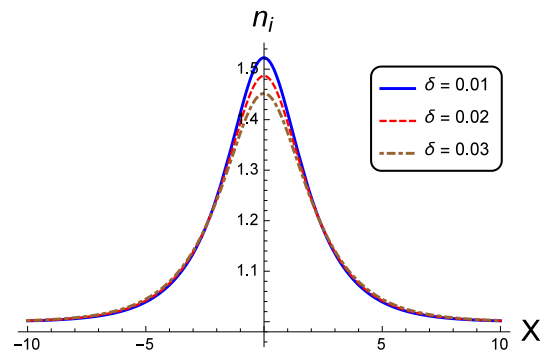

(e)

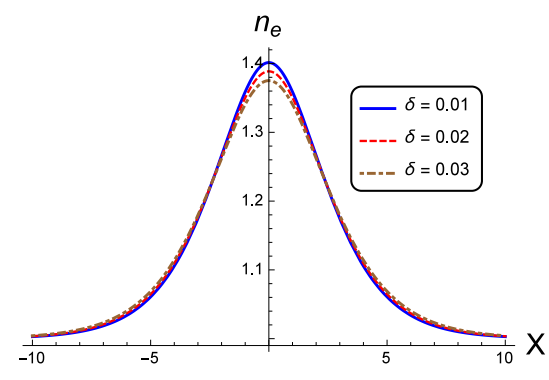

(c)

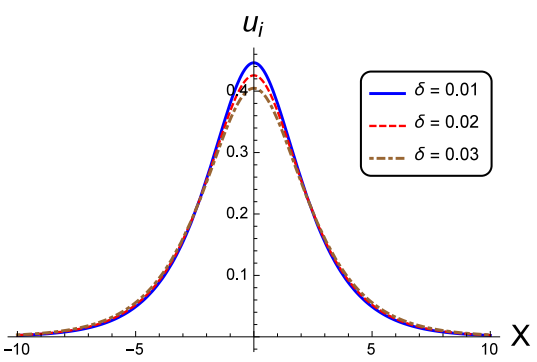

(f)

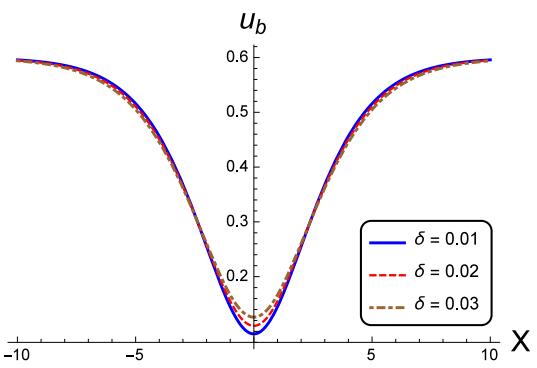

(h)

FIG. 13: (Color online) The plasma (fluid) state variables are shown in terms of the space variable $X$, for different values of the beam density (ratio) $\delta$. We have taken $V=1.3, n_{e 0}=10^{11} \mathrm{~m}^{-1}, U_{b 0}=0.6$ and $\mu_{b}=1$.

[10] Y. Nakamura, H. Bailung, and K. E. Lonngren, Phys. Plasmas 6, 3466 (1999).

[11] R. Ichiki, S. Yoshimura, T. Watanabe, Y. Nakamura, and Y. Kawai, Phys. Plasmas 9, 4481 (2002).

[12] M. Shindo and Y. Kawai, J. Phys. Soc. Jpn. 70, 621 (2001).

[13] A. J. Velasco, A. L. Chacon, W. A. Serrano, IEEE Trans Plasma Sci. 43, 1729 (2015).

[14] J. L. Cooney, D. W. Aossey, J. E. Williams, M. T. Gavin, H. S. Kim, Y. C. Hsu, A. Scheller, and K. E. Lonngren, Plasma Sources Sci. Technol.2, 73 (1993).

[15] C. Uberoi, and G. C Das, Plasma Phys. 16, 669 (1974).

[16] Yu. I. Portnyagin, O. F. Klyuev, A. A. Shidlovsky, A. N. Evdokimov, T. W. Buzdigar, P. G. Matukhin, S. G. Pasynkov, K. N. Shamshev, V. V. Sokolov, and N. D. Semkin, Adv. Space Res. 11, 89 (1991).
[17] P. H. Chaizy, H. Reme, J. A. Sauvaud, C. Duston, R. P. Lin, D. E. Larson, D. L. Mitchell, K. A. Anderson, C. W. Carlson, A. Korth, and D. A. Mendis, Nature 349, 393 (1991).

[18] T. H. Ahn, K. Nakamura, and H. Sugai, Plasma Sources Sci. Technol. 5, 139 (1996).

[19] T. Hirata, R. Hatakeyama, T. Mieno, S. Iizuka, and N. Sato, Plasma Sources Sci. Technol. 5, 288 (1996).

[20] M. J. Singh, D. Boilson, A. R. Polevoi, T. Oikawa and R. Mitteau, New J. Phys. 19, 055004 (2017).

[21] A. Y. Wong, D. L. Mamas, and D. Arnush, Phys. Fluids. 18, 1489 (1975).

[22] G. C. Das, IEEE Plasma Sci. PS-4, 199 (1976).

[23] G. O. Ludwig, J. L. Ferreira, and Y. Nakamura, Phys. Rev. Lett. 52, 275 (1984).

[24] Y. Nakamura and I. Tsukabayashi, Phys. Rev. Lett. 52, 
2356 (1984).

[25] M. Saito, S. Watanabe, and H. Tanaka, J. Phys. Soc. Jpn. 53, 2304 (1984).

[26] K.P. Das, F. Verheest, J. Plasma Phys. 41, 139 (1989).

[27] R. L. Merlino and J. J. Loomis, Phys. Fluids B 2, 2865 (1990).

[28] T. Takeuchi, S. Iizuka, and N. Sato, Phys. Rev. Lett. 80, 77 (1998).

[29] S. C. Sharma and A. Gahlot, Phys. Plasmas 15, 073705 (2008).

[30] H. Bailung, S. K. Sharma, and Y. Nakamura, Phys. Plasmas 17, 062103 (2010).

[31] S. Knist, F. Greiner, F. Biss, and A. Piel, Contrib. Plasma Phys. 51, 769 (2011).

[32] M. Rosenberg and R. Merlino, J. Plasma Phys. 79, 949 (2013).

[33] P. Pathak, S. K. Sharma, Y. Nakamura, and H. Bailung, Phys. Plasmas 23, 022107 (2016).

[34] A. Kumar and V. Mathew, Phys. Plasmas 24, 092107 (2017).

[35] D. A. Uzdensky and S. Rightley, Rep. Prog. Phys. 77, 036902 (2014)

[36] P. K. Shukla and B. Eliasson, Rev. Mod. Phys. 83, 885 (2011).

[37] V. E. Fortov, Phys. Usp. 52, 615 (2009).

[38] H. Van Horn, Physics Today 35(5), 23 (1979).

[39] G. Manfredi, Fields Inst. Commun. 46, 263 (2005).

[40] G. Manfredi and J. Hurst, Plasma Phys. Control. Fusion 57, 054004 (2015)

[41] M. E. Yahia, I. M. Azzouz, and W. M. Moslem, Applied Physics Letters 103, 082105 (2013).

[42] R. Kodama, P. A. Norreys, K. Mima, A. E. Dangor, R. G. Evans, H. Fujita, Y. Kitagawa, K. Krushelnick, T. Miyakoshi, N. Miyanaga, T. Norimatsu, S. J. Rose, T. Shozaki, K. Shigemori, A. Sunahara, M. Tampo, K. A. Tanaka, Y. Toyama, T. Yamanaka and M. Zepf, Nature
412, 798 (2001).

[43] W. Bednarek, Astrophys. J. 402, L29 (1993).

[44] W. Bednarek, J.G. Kirk, A. Mastichiadis , Astron. Astrophys. 307, L17 (1996).

[45] J. H. Beall and W. Bednarek, Astrophys. J. 510, 188 (1999).

[46] K. Sauer and E. Dubinin, Phys. Scr. T107, 167 (2004).

[47] M. E. Dieckmann, B. Eliasson, and P. K. Shukla, Phys. Rev. E 70, 036401 (2004).

[48] M. E. Dieckmann, A. Meli, P. K. Shukla, L.O.C. Drury, and A. Mastichiadis, Plasma Phys. Controlled Fusion 50, 065020 (2008)

[49] Manoj Kr. Deka, Apul N. Dev, Amar P. Misra and Nirab C. Adhikary, Phys. Plasmas 25, 012102 (2018).

[50] G. Manfredi and F. Haas, Phys. Rev. B 64, 075316 (2001).

[51] H. Bailung and S. K. Sharma, Phys. Plasmas 17, 032301 (2010); S. K. Sharma and H. Bailunga Phys. Plasmas 17, 062103 (2010).

[52] M. McKerr, F. Haas, I. Kourakis, Physical Review E, 90, 033112 (2014)

[53] I. Kourakis, M. McKerr, I.S. Elkamash and F. Haas, Plasma Physics and Controlled Fusion, 59 (10), 1050132017 (2017).

[54] I. S. Elkamash, I. Kourakis and F. Haas, Physical Review E 96, 043206 (2017)

[55] P.H. Chavanis, Phys. Rev. D 76, 023004 (2007).

[56] I.S. Elkamash, F. Haas and I. Kourakis, Physics of Plasmas, 24, 092119 (2017).

[57] F. Haas, Quantum Plasmas: An Hydrodynamic Approach, Springer (New York, 2011).

[58] A.E. Dubinov, Plasma Physics Reports, 35 (11), 991 (2009); original text published in Russian as: Fizika Plazmy, 35 (11), 1070 (2009). 